\title{
A survey of the stellar rotation in barred galaxies ${ }^{\star, \star \star}$
}

\author{
D. Bettoni ${ }^{1}$ and G. Galletta ${ }^{2}$ \\ 1 Osservatorio Astronomico, Vicolo dell' Osservatorio, 5, I-35122 Padova, Italy \\ 2 Dipartimento di Astronomia, Universitá di Padova, Vicolo dell'Osservatorio, 5, I-35122 Padova, Italy
}

Received September 18; accepted October 24, 1996

\begin{abstract}
We present stellar velocity- and velocity dispersion- data for $13 \mathrm{SB} 0$ galaxies and one SBa, based on 61 spectra collected during a long-term project developed at ESO. Our primary goal is to provide a database widest as possible for the study of the stellar velocity and velocity dispersion fields and for future modeling. New data are presented for six galaxies, while for the remaining SB0s particular kinematical aspects were already discussed in previous papers.

We found the following results: a) emission lines were detected in our spectra only for 4 out of 14 galaxies considered. When present, they reveal a very peculiar kinematics, including counter-rotation, warps or radial flows. b) A new case of gas counter-rotation has been found: NGC 7079. c) The velocity dispersion along the bar has a smoother trend than in the rest of the galaxy. However there is, in general, a similarity between the velocity dispersion trend (decreasing or flat) measured along the bar and that outside it. d) The existence of a waving pattern in the stellar rotation curves of bars is confirmed for the galaxies of our sample seen inclined between 30 and $50^{\circ}$. In addition to this effect, the percentage of non-circular stellar motions present in the barred galaxies studied may reach the $20 \%$.
\end{abstract}

Key words: galaxies: kinematics and dynamics galaxies: elliptical and lenticular

\section{Introduction}

Bars are common features of disc galaxies, happening in more than one third of all disk galaxies. Theoretical works

Send offprint requests to: D. Bettoni

* Based on observations collected at the European Southern Observatory, La Silla, Chile.

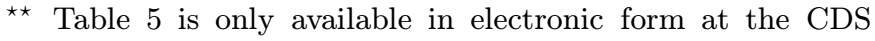
via anonymous ftp to cdsarc.u-strasbg.fr (130.79.128.5) or via http://cdsweb.u-strasbg.fr/Abstract.html on barred spirals have been successful so far in explaining several observed features: gas velocity fields and observed shapes of the bar, for instance, have been reproduced with a satisfying agreement with the observations. Observational studies of the motion of the ionized gas and of the HI are available in the literature since dozen of years (see Sellwood \& Wilkinson 1993 for a review on observations and theories).

Concerning the stellar motions, the situation for barred galaxies is quite different. Stellar orbits across and near the bar have been accurately modeled but, in spite of these progresses in the theory, few new data on the stellar velocity fields in barred spirals are available in the literature. Our knowledge of SB0, where emission lines or $21 \mathrm{~cm}$ flux are generally fainter than in spirals, is based on few, well studied objects, such as NGC 936 (Kormendy 1983) and NGC 4596 (Kent 1990). However, many of the published data concern peculiar cases, and studies of single, normal SB0s are very rare to be found in the literature. On the other side, SB0s would be ideal systems to study the structure of the bars and the stellar orbits, being more free than spiral galaxies by the presence of gas and dust.

Only quite recently, some attempts to observe the stellar kinematics of a large sample of early-type barred systems has been performed (Kormendy 1982b; Bettoni \& Galletta 1988; Jarvis et al. 1988). In these papers, the most important questions concern the dynamical interaction of the bars with the bulges and/or the halos and the three-dimensional structure of bars and ovals. The results of these studies show that stellar orbits also are influenced by the bar, with streaming motions deviating from circular orbits (Kormendy 1982a,b; Kent 1990) and with orbits sometimes retrograde (Bettoni 1989).

In order to better understand the dynamical structures present in these barred systems, we felt the need to have a set of data obtained with similar quality (velocity resolution, sky brightness, reduction methods) and for a number of barred S0s as wide as possible. As described below, the data presented here have all a velocity dispersion better than $45 \mathrm{~km} \mathrm{~s}^{-1}$ and are taken for each galaxy at least 


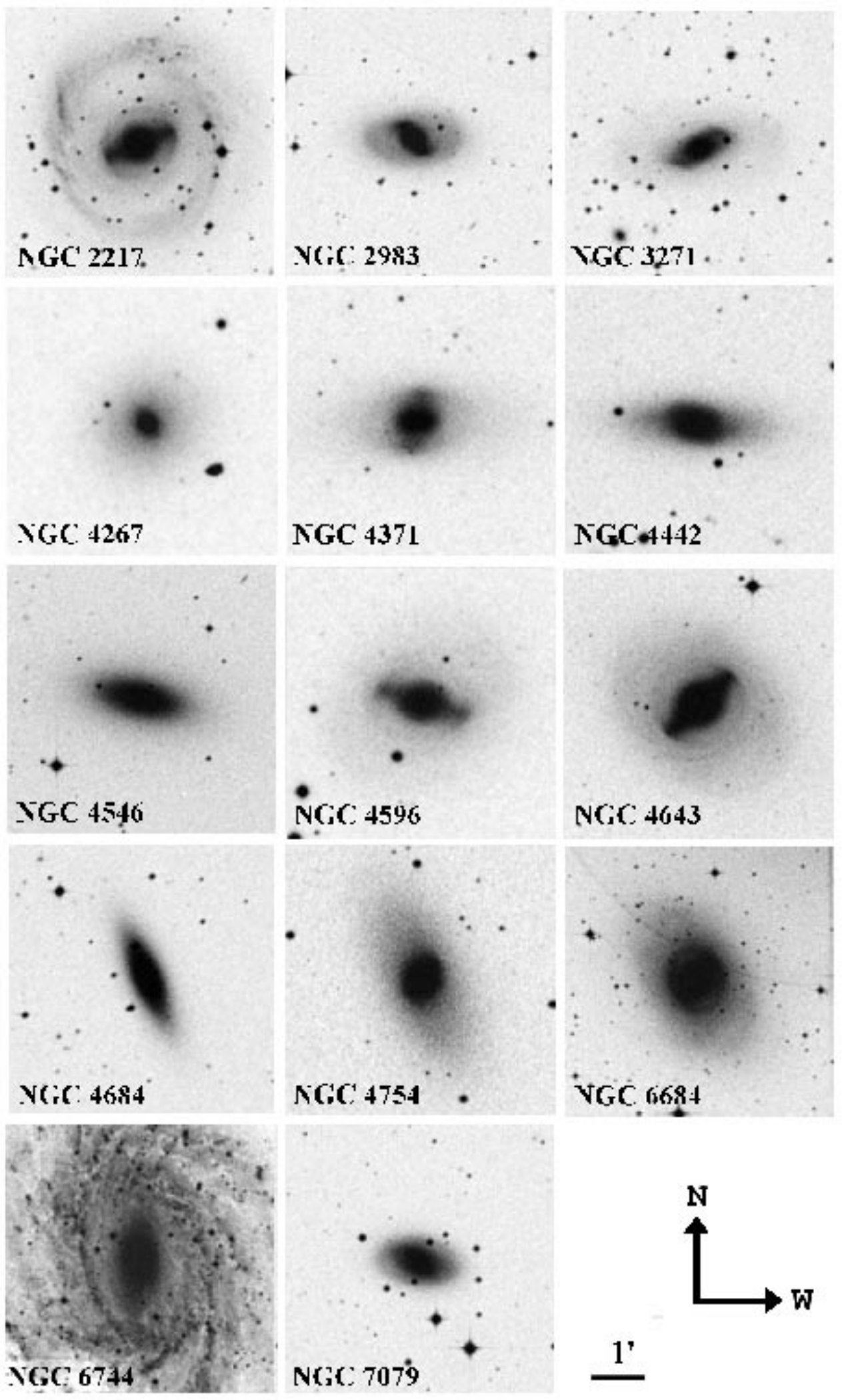

Fig. 1. Images of the studied galaxies, from Digitized Sky Survey. The field for each galaxy is $5^{\prime} \times 5^{\prime}$ 
Table 1. Log of the observations

\begin{tabular}{|c|c|c|c|c|c|c|}
\hline Date & PA & $\begin{array}{l}\text { Exp. } \\
\text { min. }\end{array}$ & seeing & $\begin{array}{c}V_{\mathrm{s}} \\
\mathrm{km} \mathrm{s}^{-1}\end{array}$ & $\begin{array}{c}\sigma_{\mathrm{s}} \\
\mathrm{km} \mathrm{s}^{-1}\end{array}$ & Notes \\
\hline \multicolumn{7}{|l|}{$N G C 3271$} \\
\hline 1989, April 1 & $106^{\circ}$ & 90 & 0.7 & $3862 \pm 16$ & $250 \pm 14$ & Major axis \\
\hline 1989, April 2 & $127^{\circ}$ & 90 & $1^{\prime \prime} 2$ & $3848 \pm 21$ & $251 \pm 36$ & Bar \\
\hline 1989, April 3 & $16^{\circ}$ & 90 & $1^{\prime \prime} 0$ & $3770 \pm 14$ & $211 \pm 22$ & Minor axis \\
\hline 1989, April 5 & $61^{\circ}$ & 90 & 1.. 1 & $3833 \pm 15$ & $249 \pm 24$ & \\
\hline \multicolumn{7}{|l|}{$N G C 4371$} \\
\hline 1988, March 15 & $95^{\circ}$ & 90 & 1.1 & $912 \pm 5$ & $138 \pm 3$ & Major axis \\
\hline 1988, March 15 & $165^{\circ}$ & 90 & $1 . .2$ & $900 \pm 5$ & $140 \pm 5$ & Bar \\
\hline 1988, March 15 & $50^{\circ}$ & 90 & $1^{\prime \prime} 0$ & $914 \pm 5$ & $139 \pm 6$ & \\
\hline 1988, March 15 & $140^{\circ}$ & 90 & $1^{\prime \prime} 1$ & $930 \pm 5$ & $138 \pm 4$ & \\
\hline \multicolumn{7}{|l|}{$N G C 4596$} \\
\hline 1989, April 1 & $135^{\circ}$ & 120 & $0 . .7$ & $1908 \pm 14$ & $149 \pm 8$ & Major axis \\
\hline 1989, April 1 & $72^{\circ}$ & 90 & $1^{\prime \prime} 5$ & $1892 \pm 10$ & $157 \pm 8$ & Bar \\
\hline 1989, April 1 & $45^{\circ}$ & 90 & $1^{\prime \prime} 5$ & $1883 \pm 11$ & $157 \pm 9$ & Minor axis \\
\hline 1989, April 1 & $0^{\circ}$ & 49 & $1 . .5$ & $1904 \pm 12$ & $145 \pm 3$ & \\
\hline \multicolumn{7}{|l|}{$N G C 4754$} \\
\hline 1989, April 3 & $23^{\circ}$ & 120 & $1^{\prime \prime} .0$ & $1302 \pm 12$ & $191 \pm 4$ & Major axis \\
\hline 1989, April 3 & $141^{\circ}$ & 90 & $1 . .0$ & $1317 \pm 1$ & $194 \pm 8$ & Bar \\
\hline 1989, April 3 & $68^{\circ}$ & 69 & $1^{\prime \prime} 0$ & $1340 \pm 9$ & $183 \pm 13$ & \\
\hline 1989, April 5 & $113^{\circ}$ & 90 & 1.. 3 & $1336 \pm 6$ & $186 \pm 7$ & Minor axis \\
\hline \multicolumn{7}{|l|}{$N G C 6744$} \\
\hline 1986, May 4 & $105^{\circ}$ & 90 & $1 .{ }^{\prime \prime} 0$ & $910 \pm 8$ & $117 \pm 28$ & Minor axis \\
\hline 1986, May 6 & $15^{\circ}$ & 120 & $1^{\prime \prime} 5$ & $890 \pm 6$ & $112 \pm 25$ & Major axis \\
\hline 1986, May 6 & $60^{\circ}$ & 90 & $1^{\prime \prime} .5$ & $940 \pm 8$ & $115 \pm 33$ & \\
\hline 1986, May 6 & $0^{\circ}$ & 90 & $1^{\prime \prime} 5$ & $840 \pm 23$ & $110 \pm 30$ & Bar \\
\hline \multicolumn{7}{|l|}{ NGC 7079} \\
\hline 1987, May 2 & $82^{\circ}$ & 120 & $1^{\prime \prime} .0$ & $2729 \pm 7$ & $141 \pm 11$ & Major axis \\
\hline 1987, May 3 & $39^{\circ}$ & 90 & $1^{\prime \prime} 0$ & $2648 \pm 7$ & $159 \pm 3$ & Bar \\
\hline 1987, Мay 3 & $170^{\circ}$ & 90 & $1^{\prime \prime} 5$ & $2674 \pm 7$ & $175 \pm 12$ & Minor axis \\
\hline 1987, May 3 & $127^{\circ}$ & 75 & $1^{\prime \prime} 5$ & $2659 \pm 7$ & $171 \pm 11$ & \\
\hline
\end{tabular}

at four different position angles on the sky, to study the velocity field of the single galaxies. We also have the goal of providing homogeneous and detailed new data for the future models of barred galaxies.

In this paper we present new velocity and velocity curves for six galaxies (five SB0 and one SBa, NGC 3271, NGC 4371, NGC 4596, NGC 4754, NGC 6744 and NGC 7079), and we analyze the original data for 8 additional cases, observed with similar instruments, but discussed in previous studies. In Sect. 2 we give an overview of the observations; in Sect. 3 we describe the observed characteristics of each galaxy and finally in Sect. 3 the main results of this study.

\section{The sample galaxies}

\subsection{Observations and data reduction}

The observations were obtained, as part of a long term program, during four different observing runs from 1986 to 1989 with the ESO/MPI 2.2-m telescope at La Silla, using the Cassegrain Boller \& Chivens spectrograph. In May 1986 and May 1987 we used a $320 \times 512$ pixel RCA CCD and a dispersion of $1.77 \AA$ pixel $^{-1}$; the scale perpen-

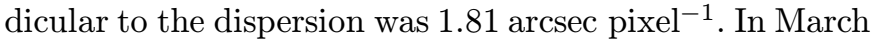
1988 and April 1989 a $640 \times 1024$ pixel RCA CCD was available, giving a better dispersion $\left(0.885 \AA\right.$ pixel $\left.^{-1}\right)$ and an higher scale perpendicular to the dispersion $(0.885$ arcsec pixel $\left.^{-1}\right)$. In all the cases the interval studied was $\lambda \lambda$ $4900-5700 \AA$ and the slit width was set to $1.5 \operatorname{arcsec}$ on the sky. The first set of data allows a velocity dispersion resolution $\Delta \sigma=43 \mathrm{~km} \mathrm{~s}^{-1}$ while the second one has $\Delta \sigma=42 \mathrm{~km} \mathrm{~s}^{-1}$. In addition to the galaxy spectra, every observing night the spectrum of at least one giant $\mathrm{K}$ star of low rotational velocity, $v \sin i<17 \mathrm{~km} \mathrm{~s}^{-1}$, was recorded for use as a template of zero velocity dispersion.

Details of the selected position angles and exposure times are given in Table 1. In Cols. 1, 2 and 3 we give the observing date, the position angle and the exposure time 
of each spectrum. In Col. 4 we report the seeing present during each exposure. In Cols. 5 and 6 we give the systemic velocity corrected to the sun and the central velocity dispersion (averaged over the central 3 arcsec).

All the spectra obtained have been reduced by means of the ESO-IHAP procedures and then analyzed using the FQ method (Bertola et al. 1984) generating the stellar rotation and velocity dispersion curves reproduced in Figs. 1-5.

\subsection{Description of the galaxies}

In the following, the principal characteristics of the galaxies are summarized. The values reported for the velocity dispersion and for the systemic velocity are computed from the mean of the different spectra. Our systemic velocities are generally in agreement with the values reported by $\mathrm{RC} 3$. The greatest difference we found is $\sim 100 \mathrm{~km} \mathrm{~s}^{-1}$ for NGC 4754 .

Their observed properties are listed in Table 2, for the discussion in Sect. 3.

\section{NGC 3271}

This galaxy is classified SB0(r) in RC3 (de Vaucouleurs et al. 1991) and Sa by Sandage \& Bedke (1995) (RSA). The optical image show a quite thick bar, extending $32^{\prime \prime}$, a quite high percentage of the disk. At the ends of the bar, two dust lanes mark two incipient spiral arms (Fig. 1).

In the literature, no kinematical data are available. The bar is along $\mathrm{PA}=127^{\circ}, \sim 30^{\circ}$ from the apparent major axis of the galaxy. From our data we derive a maximum rotational velocity of $\sim 150 \mathrm{~km} \mathrm{~s}^{-1}$ along the major axis. The velocity dispersion has a very pronounced maximum of $\sim 250 \mathrm{~km} \mathrm{~s}^{-1}$ in the nucleus. This peaked trend appears in all the four position angles studied. The velocity dispersion decrease to $\sim 100 \mathrm{~km} \mathrm{~s}^{-1}$ in the outer regions $\left(r>10^{\prime \prime}\right)$ on both sides of the galaxy, where the disk dominates.

\section{NGC 4371}

This galaxy has been classified $\mathrm{SB} 0(\mathrm{r})+$ by $\mathrm{RC} 3$ and $\mathrm{SB0}_{2 / 3}(\mathrm{r}) 3$ by $\mathrm{RSA}$. The bar is clearly visible along the apparent minor axis of the disk. It has been studied also by Kormendy (1982a) by means of a spectrum at PA = $90^{\circ}$ ( $5^{\circ}$ from major axis). He found a central velocity dispersion $\sigma=106 \mathrm{~km} \mathrm{~s}^{-1}$ and a maximum of rotation of $125 \mathrm{~km} \mathrm{~s}^{-1}$. From our data, taken at $\mathrm{PA}=95^{\circ}$, we measure a higher rotational velocity $V_{\max } \sim 190 \mathrm{~km} \mathrm{~s}^{-1}$ and also a slightly higher velocity dispersion $\sigma=139 \pm$ $2 \mathrm{~km} \mathrm{~s}^{-1}$.

\section{NGC 4596}

This is a well known example of SB0 galaxy; SB0(r)+ in RC3. It has been yet classified as SBa(very early) in RSA. Photometry and kinematics of the bar have been studied by Kent (1990), who found that the bar in this galaxy is roughly rectangular, and that the kinematics of the bar do not differ from the mean rotation of the disk of the galaxy. The rotation curve along the bar shows a peak of velocity near the center, followed by a decrease and by a new rise outward. This shape is confirmed by our measurements and it appears to be another case of the waving rotation curve phenomenon observed in the bars of many SB0s (Bettoni 1989). The velocity dispersion is almost constant along all the observed PAs and our value (Table 2) is in very good agreement with previous published data (Mcelroy 1995).

\section{NGC 4754}

This barred $\mathrm{S} 0$ is classified SB0(r)- in RC3 and $\mathrm{SB}_{1}(\mathrm{~s})$ in RSA. The bar is not very strong and is confined in the inner regions of the bulge of the galaxy. The kinematics along the major and minor axes of the bar in this galaxy has been studied by Jarvis et al. (1988). They found a velocity gradient of $100 \mathrm{~km} \mathrm{~s}^{-1}$ along both axes. Our data along $\mathrm{PA}=141^{\circ}$ (bar major axis) are in good agreement. NGC 6744

This extended barred spiral galaxy is classified $\mathrm{SAB}(\mathrm{r}) \mathrm{bc}$ in RC3 and $\mathrm{Sbc}(\mathrm{r}) \mathrm{II} / \mathrm{SBbc}(\mathrm{r}) \mathrm{II}$ in RSA. This spiral galaxy has been included in our sample of SB0 because the region dominated by the bar is very extended and quite clean from gas. The results can be then easily compared with the more classical SB0 galaxies. The only kinematical data available for this galaxy are from $\mathrm{HI}$ observations (Whiteoak \& Gardner 1977). The HI offsets pointing along the major axis shows the two-horned profile typical of spiral galaxies, a maximum of $\sim 166 \mathrm{~km} \mathrm{~s}^{-1}$ is reached at 18 arcmin. Our measured stellar rotation curve, in the central 3 arcmin, is in agreement with the HI data and reaches a maximum of rotation of $\sim 144 \mathrm{~km} \mathrm{~s}^{-1}$. No emission lines are visible in our green spectra, but VeronCetty \& Veron (1986) report the presence of [NII] and $\mathrm{H} \alpha$.

\section{NGC 7079}

This galaxy is a member of a group (Garcia 1993) and is a member of a physical pair (\# 351, Reduzzi \& Rampazzo 1995), it has been classified as SB0(s)0 by RC3 and SBa by RSA. The bar in this galaxy is not very strong.

For this galaxy Bettoni \& Buson (1987) indicate the presence of faint emission lines; in our spectra it has been possible to measure only the $\mathrm{O}[\mathrm{III}] \lambda=5007 \AA$ line, in the nuclear region within 15 arcsec. From our measurements we found that the gas is in counter-rotation with respect to the stars along all the four observed position angles. Probably we are observing a very small disk of gas, whose maximum extension is $\sim 15^{\prime \prime}$ (along the apparent major axis).

\subsection{Expanding the database}

Together with the new data discussed in the previous paragraph, we have included in this paper the velocity curves of 8 more galaxies, observed at La Silla with the same spectrograph, within the same long term project. They are: NGC 2217 (Bettoni et al. 1990), NGC 2983 (Bettoni et al. 1988), 

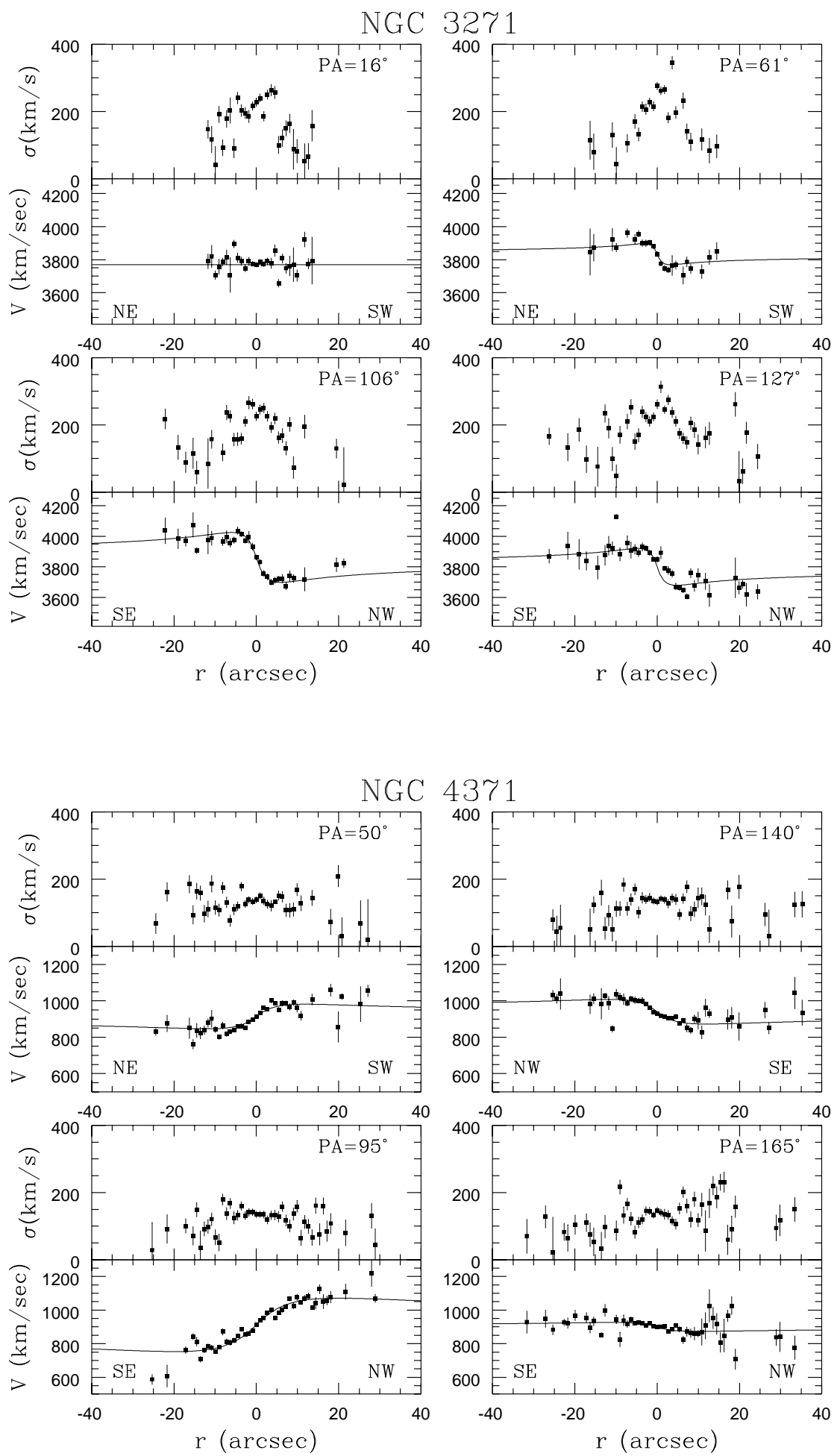

Fig. 2. Velocity dispersion $(\sigma)$ and Radial Velocity $(V)$ curves for the six galaxies for which new data are presented. All the velocities are heliocentric. The continuum line is the model velocity, as explained in the text 

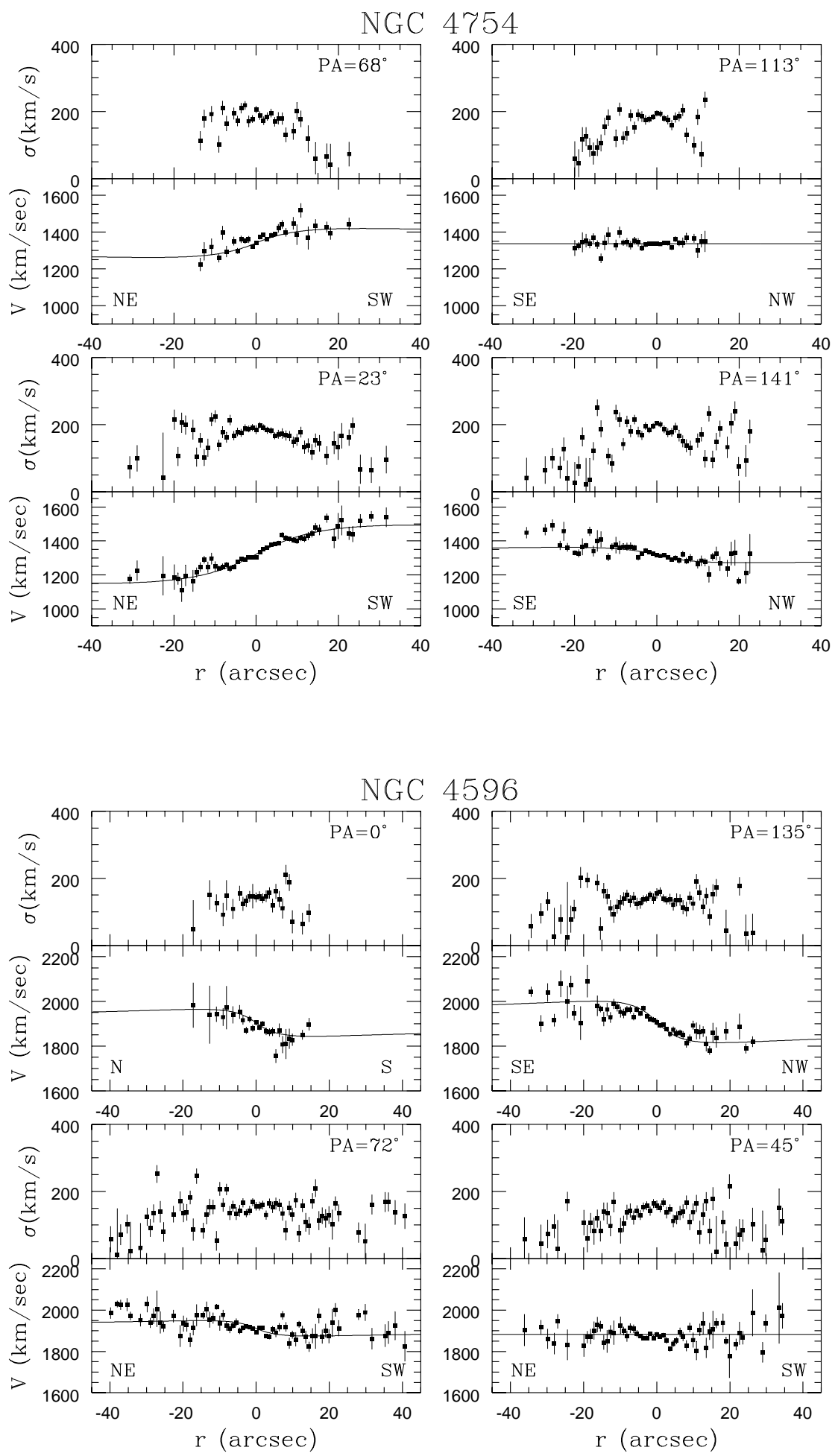

Fig. 3. As in the previous figure 

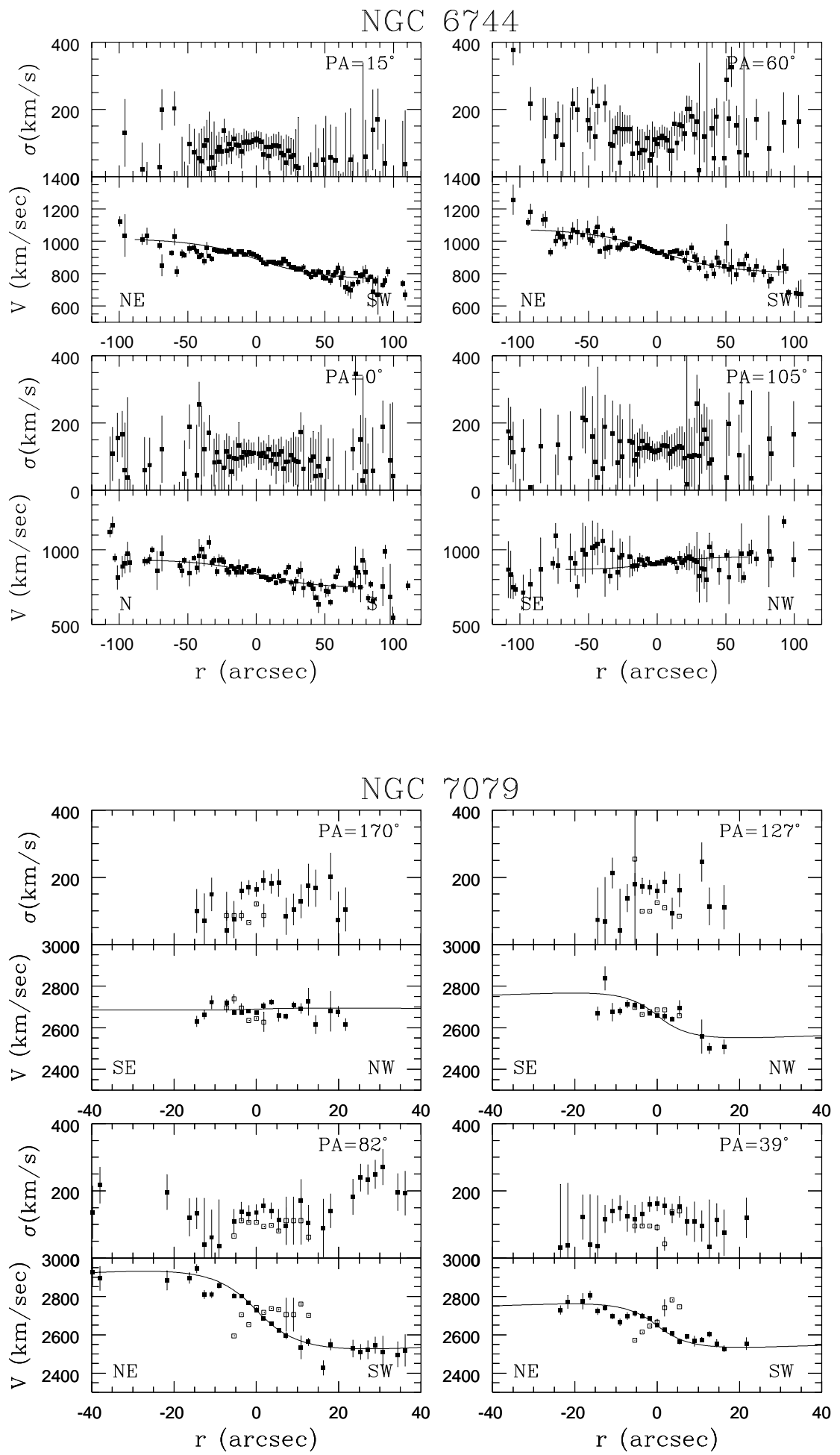

Fig. 4. As in the previous figure 

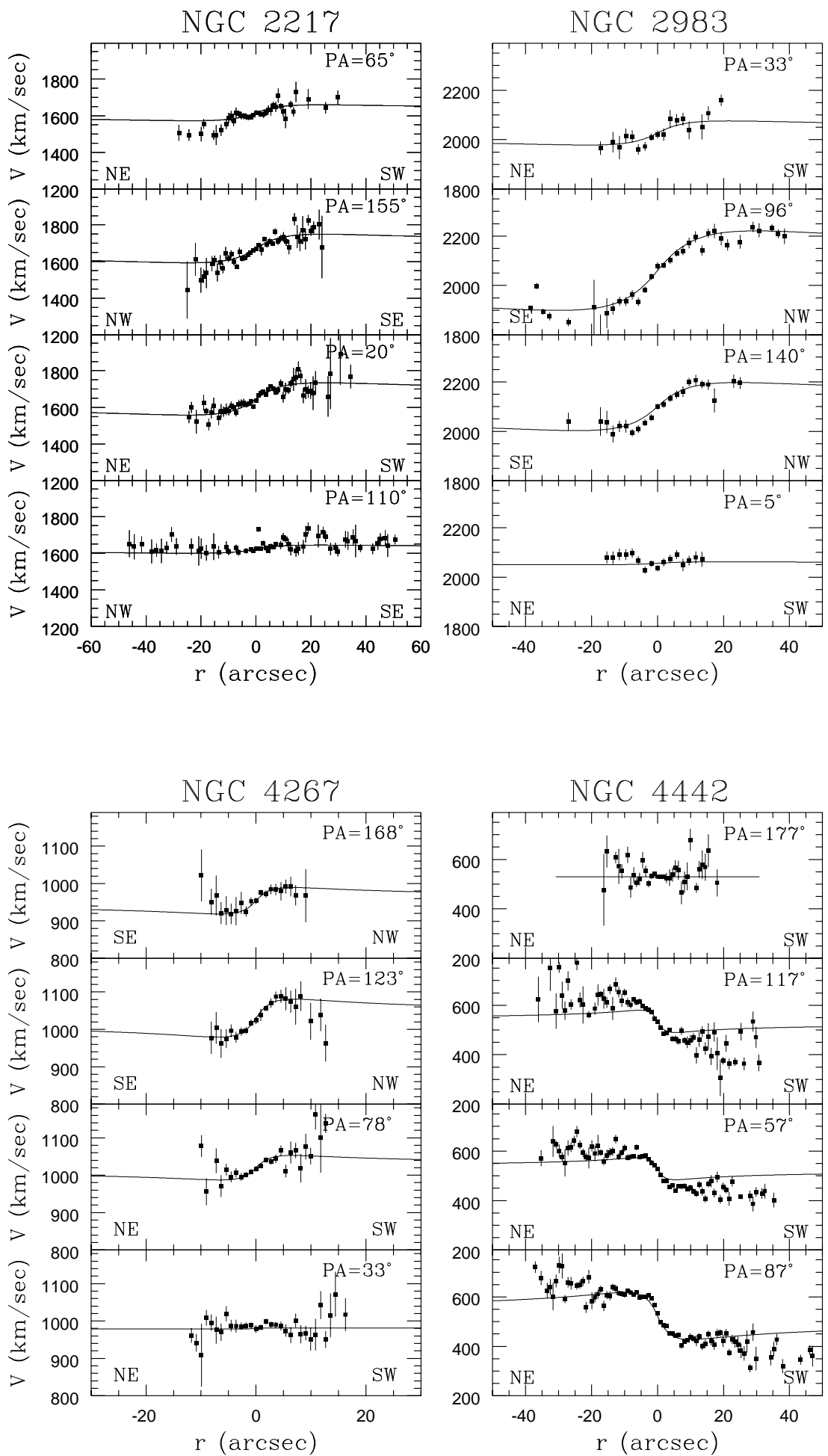

Fig. 5. Rotation curves of galaxies included in the samples, fitted with a model of circular rotation on the galaxy plane. See the text for more details 


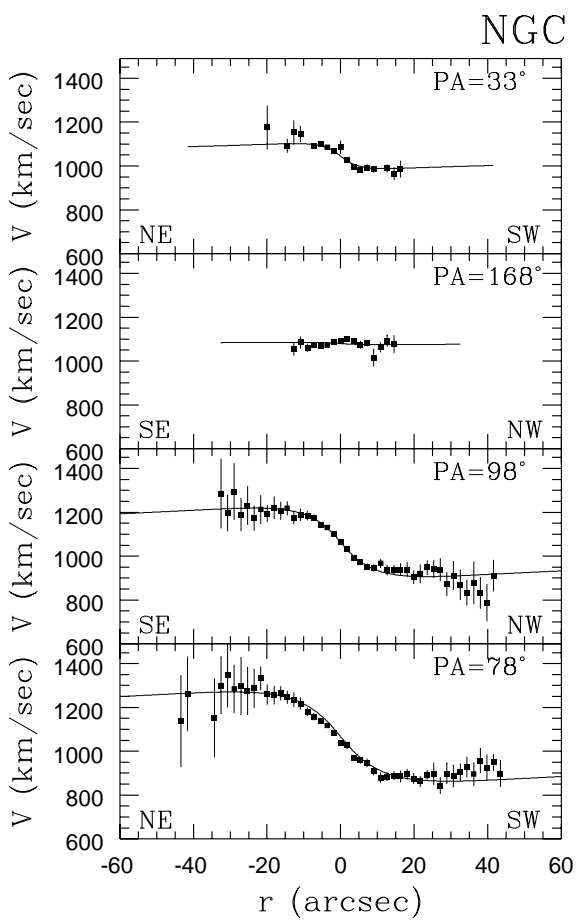

4546
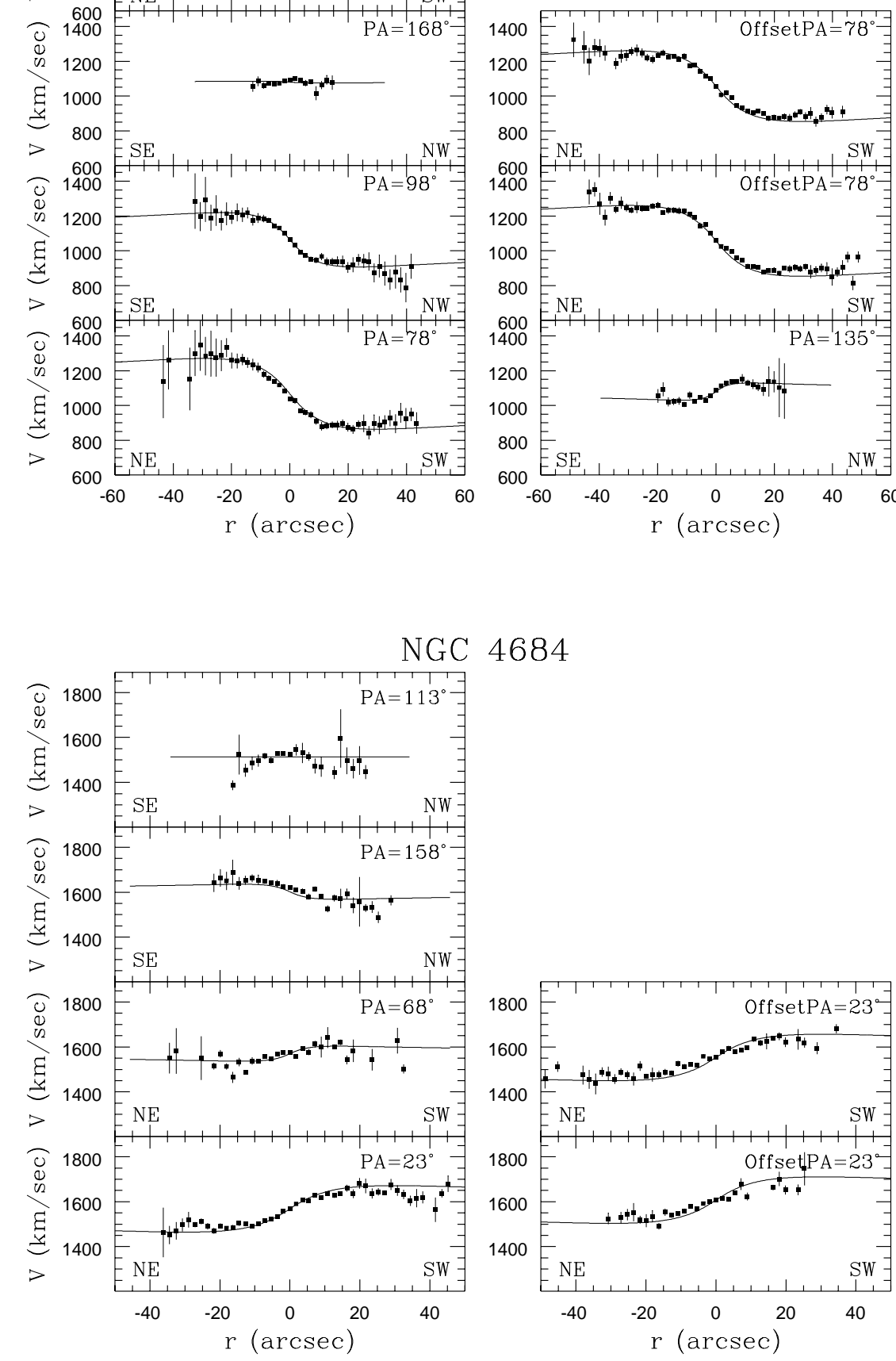

4684

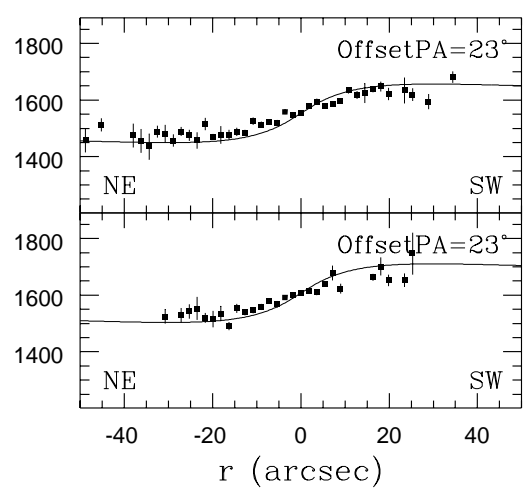

Fig. 6. As in the previous figure 

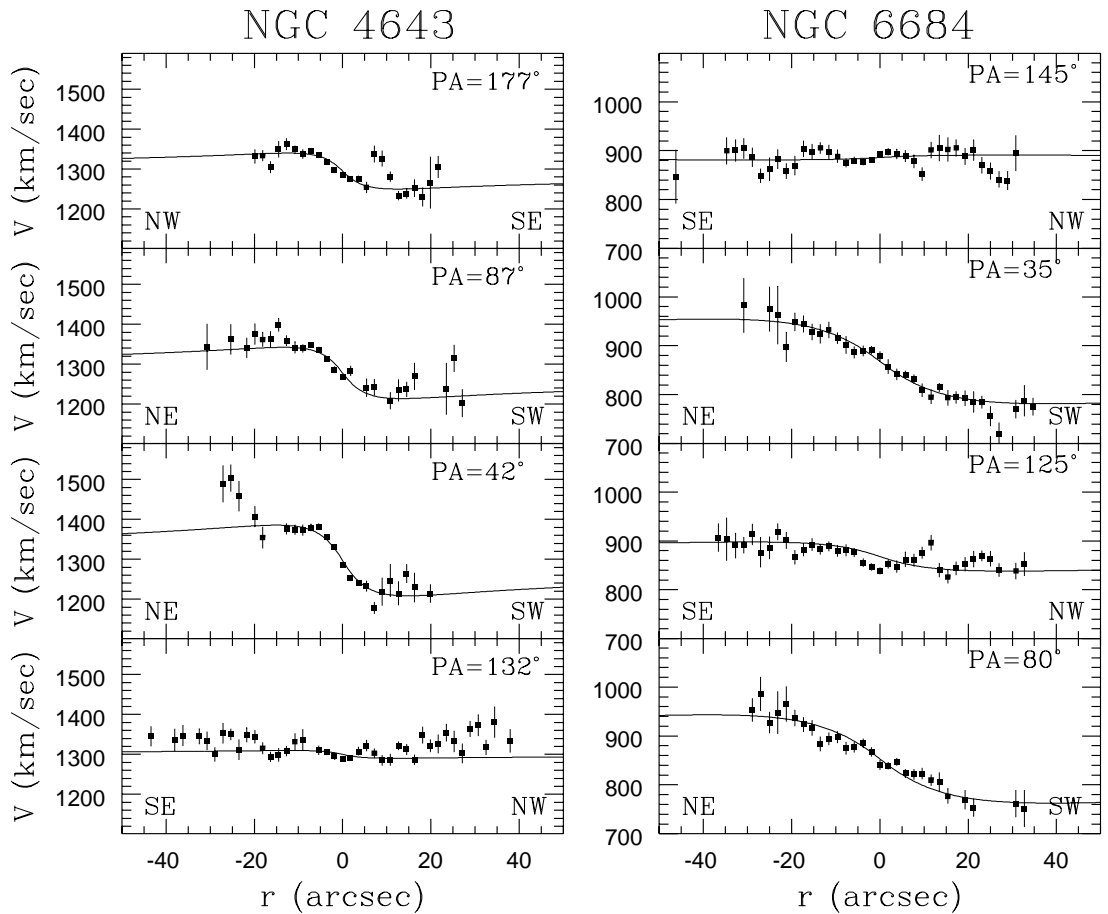

Fig. 7. As in the previous figure

Table 2. Observed properties of sample galaxies. The systemic velocity $c z$ and the central velocity dispersion are extracted by the detailed studies on the single galaxies cited in the text. Galaxy inclinations $i$ are deduced assuming an intrinsic axial ratio 0.25

\begin{tabular}{|c|c|c|c|c|c|c|c|c|c|c|c|}
\hline \multirow[b]{2}{*}{ NGC } & \multirow[b]{2}{*}{ Type $^{\dagger}$} & \multirow[b]{2}{*}{$B_{\mathrm{T}}^{\dagger}$} & \multirow[b]{2}{*}{$\begin{array}{r}c z \\
\mathrm{~km} / \mathrm{s}\end{array}$} & \multirow[b]{2}{*}{$\begin{array}{r}\sigma \\
\mathrm{km} / \mathrm{s}\end{array}$} & \multicolumn{4}{|c|}{ Disk } & \multicolumn{3}{|c|}{ Bar } \\
\hline & & & & & $\mathrm{PA}_{0}^{\dagger}$ & $D_{25}^{\dagger}$ & $b / a^{\dagger}$ & $\begin{array}{l}i \\
\circ\end{array}$ & $\begin{array}{r}\mathrm{PA} \\
0\end{array}$ & $\underset{\prime \prime}{D}$ & $D_{\text {॥ }}$ \\
\hline$\overline{2217}$ & $\mathrm{RLBT}+$ & 11.7 & 1636 & 221 & 10 & 268 & 0.93 & 22 & 111 & 90 & 97 \\
\hline 2983 & $\mathrm{LBT}+$ & 12.8 & 2063 & 163 & 95 & 151 & 0.59 & 57 & 38 & 38 & 61 \\
\hline 3271 & LBR0 & 12.9 & 3828 & 240 & 106 & 185 & 0.59 & 57 & 122 & 64 & 69 \\
\hline 4267 & LBS- & 11.9 & 972 & 168 & 130 & 194 & 0.93 & 22 & 35 & 40 & 43 \\
\hline 4371 & $\mathrm{LBR}+$ & 11.8 & 914 & 139 & 95 & 239 & 0.56 & 59 & 163 & 65 & 119 \\
\hline 4442 & LBS0 & 11.4 & 529 & 192 & 87 & 274 & 0.39 & 72 & 69 & 43 & 59 \\
\hline 4546 & LBS-* & 11.3 & 1069 & 242 & 78 & 199 & 0.44 & 68 & 47 & 41 & 67 \\
\hline 4596 & $\mathrm{LBR}+$ & 11.4 & 1897 & 152 & 135 & 239 & 0.74 & 44 & 70 & 101 & 134 \\
\hline 4643 & SBT0 & 11.7 & 1259 & 163 & 48 & 185 & 0.74 & 44 & 134 & 101 & 140 \\
\hline 4684 & LBR+ & 12.3 & 1578 & 80 & 23 & 173 & 0.36 & 75 & 158 & 52 & 142 \\
\hline 4754 & LBR-* & 11.5 & 1324 & 189 & 23 & 274 & 0.54 & 60 & 143 & 64 & 117 \\
\hline 6684 & PLBS0 & 11.3 & 866 & 93 & 35 & 239 & 0.66 & 51 & 150 & 62 & 93 \\
\hline 6744 & SXR4 & 9.1 & 895 & 114 & 15 & 1197 & 0.65 & 52 & 0 & 142 & 149 \\
\hline 7079 & LBS0 & 12.5 & 2678 & 159 & 82 & 128 & 0.62 & 54 & 39 & 42 & 58 \\
\hline
\end{tabular}

$\dagger$ from RC3. 
NGC 6684 (Bettoni \& Galletta 1988), NGC 4546 (Galletta 1987; Bettoni et al. 1991), NGC 4267, NGC 4643 (Magrelli et al. 1992), NGC 4684 (Bettoni et al. 1993) and NGC 4442 (Bettoni \& Galletta 1994). The above galaxies have been observed with the same telescope and spectrograph (2.2 m ESO- MPI + B\&C) and have the same quality of the previous data. The only exception are NGC 2983 and NGC 6684 that have been observed with the $\mathrm{B} \& \mathrm{C}$ spectrograph mounted at the Cassegrain focus of the $3.6 \mathrm{~m}$ ESO telescope. For these latter the velocity resolutions $\left(37 \mathrm{~km} \mathrm{~s}^{-1}\right)$ and the scale (1.93 arcsec/pixel) are similar. Due to particular features present in these galaxies (counter-rotation, filaments, bar asymmetries), part of their kinematical data have been already published. A description of the main photometric and kinematical properties of these galaxies has been reported in the corresponding papers. In Table 5 are reported the kinematical data for the sample galaxies. Radii are in $\operatorname{arcsec} ; V$ and $\sigma$ are in $\mathrm{km} / \mathrm{s}$. These data are plotted in Figs. 2-7.

\section{Discussion}

The main properties of the whole sample galaxies are presented in Table 2. The sample includes systems in a wide range of apparent inclinations, from nearly face-on objects (NGC 2217, $i=22^{\circ}$ ) to galaxies seen almost edge-on $\left(\mathrm{NGC} 4442, i=72^{\circ}\right)$. These galaxies have been selected from RC3 among the SB0 with $m_{B}<13$ and $\delta<-20^{\circ}$.

As said in the introduction, our main purpose is to collect and make available a wide set of data on stellar kinematics of barred S0 galaxies. This data archive could be very useful to produce in the future more realistic models of stellar bars. We can, however, discuss here the main properties and problems encountered in analyzing our data.

\subsection{Peculiarities of ionized gas}

In the sample of 14 galaxies considered in this paper only 4 show the presence of [OIII] or $\mathrm{H} \beta$ emission lines: NGC 2217, NGC 4546, NGC 4684 and NGC 7079. This low percentage is in agreement with the fact that earlytype galaxies does not show the presence of ionized gas with high frequency (Bregman et al. 1992). It is interesting to note yet that in all the cases where emission lines were detectable, the gas kinematics appears decoupled from that of the stars. In two cases (NGC 4546 and NGC 7079) the gas is rotating in a direction opposite to that of the stars (gas counter-rotation). This feature, revealed in dozen of galaxies (see Galletta 1996 for a review), is generally attributed to the accretion of gas from outside. An anomalous gas structure has been also detected in NGC 2217; there, the ionized gas seems to rotate in a warped disk whose inner part is perpendicular to the bar, while the outer part lies on the main galaxy disk. Finally, in NGC 4684 strong non-circular motions are observed, with ionized gas in a thick central disk from where two opposite filaments arose, with radial motions.

This peculiarity of gas motions is quite singular; due to the small number of objects involved, one cannot say if there is a connection between these peculiarities and the presence of a bar. The highly warped gas disk of NGC 2217 may have an internal origin. Friedly \& Benz (1993) suggested that this gas disk may be modeled by vertical resonances driven by the bar.

Table 3. Deduced properties of sample galaxies

\begin{tabular}{|c|c|c|c|c|c|c|c|}
\hline$\overline{\mathrm{NGC}}$ & $\frac{R_{\mathrm{Bar}}}{\mathrm{R}_{25}}$ & $R_{\mathrm{m}}$ & $\begin{array}{r}V_{\mathrm{m}} \\
\mathrm{km} / \mathrm{s}\end{array}$ & $\underset{\mathrm{o}}{\phi_{\mathrm{n}}}$ & $\begin{array}{l}i \\
\circ\end{array}$ & $\psi$ & $\frac{\Delta V_{\text {obs }}}{V_{*}}($ bar $)$ \\
\hline$\overline{2217}$ & 0.36 & 25 & 250 & 5 & 22 & 75 & $20 \%$ irregular \\
\hline 2983 & 0.41 & 30 & 220 & 98 & 47 & 69 & waving pattern \\
\hline 3271 & 0.37 & 6 & 180 & 106 & 65 & 34 & $10 \%$ \\
\hline 4267 & 0.22 & 7 & 120 & 125 & 25 & 90 & irregular \\
\hline 4371 & 0.50 & 20 & 180 & 95 & 62 & 89 & $0 \%$ \\
\hline 4442 & 0.22 & 10 & 100 & 87 & 72 & 46 & cylindric \\
\hline 4546 & 0.33 & 30 & 220 & 79 & 71 & 62 & $10 \%$ \\
\hline 4596 & 0.56 & 17 & 180 & 135 & 31 & 68 & $10 \%+$ wav. pat. \\
\hline 4643 & 0.76 & 15 & 140 & 50 & 40 & 85 & waving pattern \\
\hline 4684 & 0.82 & 30 & 110 & 23 & 70 & 71 & $10 \%$ \\
\hline 4754 & 0.49 & 40 & 200 & 23 & 59 & 73 & $10 \%$ \\
\hline 6684 & 0.39 & 42 & 140 & 59 & 48 & 89 & waving pattern \\
\hline 6744 & 0.12 & 125 & 140 & 15 & 51 & 23 & $20 \%$ \\
\hline 7079 & 0.45 & 27 & 260 & 82 & 51 & 56 & waving pattern \\
\hline
\end{tabular}

\subsection{Waving rotation curve of stars in bars}

In five of the galaxies the rotation curve along the bar, when folded about the center of symmetry, show a "waving pattern" (see Bettoni 1989 for a discussion of the feature). The galaxies are: NGC $2983\left(\mathrm{PA}=33^{\circ}\right)$, NGC 6684 $\left(\mathrm{PA}=145^{\circ}\right), \mathrm{NGC} 7079\left(\mathrm{PA}=39^{\circ}\right), \mathrm{NGC} 4596(\mathrm{PA}=$ $\left.72^{\circ}\right)$, and NGC $4643\left(\mathrm{PA}=132^{\circ}\right)$. For NGC 4596, this deviation appears also in the data of Kent (1990). Other SB0s not included in our sample but where waving shape is present are IC 456 (Bettoni 1989), NGC 936 (Kormendy 1983), NGC 1543 and NGC 4477 (Jarvis et al. 1988). This deviation appears superimposed to the general galaxy rotation, and it is confined to the region dominated by the bar.

The waving pattern in the rotation curve has been always detected in galaxies seen at intermediate inclination (between $\sim 30^{\circ}$ and $50^{\circ}$ ). The spiral NGC 6744 is close to this empirical upper limit but has no waving pattern, while the SB0 NGC 7079, with apparently the same inclination, have it. The lack of the waving pattern effect in galaxies with low inclination suggests that the motions responsible of this peculiarity predominate on the galaxy plane 
and have no vertical components. On the other hand, inside edge-on galaxies this effect may be submerged by the predominance of the disk dynamics.

The observed amplitude of the waving pattern is quite low, $\leq 30 \mathrm{~km} \mathrm{~s}^{-1}$ and projected on the galaxy plane corresponds to non-circular deviation of $30-50 \mathrm{~km} \mathrm{~s}^{-1}$ in radial direction. A theoretical interpretation of this feature has been recently presented by Wozniak \& Pfenniger (1996) using self-consistent models. They conclude that the waving pattern is due to the presence of retrograde orbits whose amount in the galaxy can vary from 14 to $30 \%$.

Table 4. Velocity dispersion trends with the radius measured along the galaxy major axis and along the bars

\begin{tabular}{lll}
\hline NGC & $\begin{array}{l}\text { Bar } \\
\text { trend }\end{array}$ & $\begin{array}{l}\text { Maj. axis } \\
\text { trend }\end{array}$ \\
\hline 2217 & decreasing & decreasing \\
2983 & decreasing & constant \\
3271 & decreasing & decreasing \\
4267 & decreasing & decreasing \\
4371 & constant & decreasing \\
4442 & decreasing & decreasing \\
4546 & decreasing & constant \\
4596 & constant & constant \\
4643 & constant & constant \\
4684 & constant & constant \\
4754 & decreasing & decreasing \\
6684 & decreasing & decreasing \\
6744 & constant & constant \\
7079 & constant & constant \\
\hline
\end{tabular}

\subsection{Velocity dispersion in bars}

The central value of the velocity dispersion is indicated in Table 2, mediated in the innermost $3^{\prime \prime}$. One goes from "warm" systems, with $\sigma \sim 250 \mathrm{~km} \mathrm{~s}^{-1}$, to quite "cold" bulges, with values lower than $100 \mathrm{~km} \mathrm{~s}^{-1}$. Inside the galaxy, the shape of the velocity dispersion profiles vary from almost flat (e.g. NGC 4596) to sharply falling with increasing radius (e.g. NGC 3271). Table 4 indicates the observed trend of $\sigma$ measured along the bar and outside the bar (typically along the galaxy major axis). From these value we note that there is an agreement between the trend measured along the bar and the global trend in the galaxy. When a plateau of velocity dispersion is present, it is detected also outside the bar. The only exception seems to be NGC 4371, with a quite constant bar velocity dispersion, compared with a $\sigma$ decreasing with radius in the rest of the galaxy. There is, however, a tendency of $\sigma$ profiles to be more slowly decreasing inside the bar than outside.

\subsection{Deviation from circular motions}

We can suspect that a non-asymmetric structure such as the bar should modify the global galaxy rotation, introducing non-circular motions. These deviations are observed in gas kinematics, both from HI- and optical emission- data, generating an S-shape of the zero-velocity line in the velocity fields (see Peterson et al. 1978; Peterson $\&$ Huntley 1980; Huntley 1978). In our galaxies, we are interested to test the amplitude of the deviations from circularity present in the stellar velocity fields. To this purpose, we adopted a simple model, as described in the following.

Every point on the plane of the galaxy is seen at a projected distance from the center equal to:

$$
r=R \sqrt{\cos ^{2}\left(\phi-\phi_{\mathrm{n}}\right)+\sin ^{2}\left(\phi-\phi_{\mathrm{n}}\right) \cos ^{-2} i},
$$

being $\phi$ the position angle on the sky formed by a line crossing the point and starting from the center. $R$ is the true distance on the galaxy plane and $\phi_{\mathrm{n}}$ the PA of the line-of-nodes. The angular distance of the bar from the line-of-nodes on the galaxy plane is then

$\psi \simeq\left|\arctan \frac{\tan \left(\phi_{\mathrm{b}}-\phi_{\mathrm{n}}\right)}{\cos i}\right|$,

with $\phi_{\mathrm{b}}$ the bar position angle.

We can describe the observed rotation curve along the line-of-nodes with the equation:

$v\left(r, \phi_{\mathrm{n}}\right)=\frac{A R}{1+(B R)^{3 / 2}} \cdot \sin (i)$,

where $i$ is the galaxy inclination with respect to the sky plane and $A, B$ parameters of the curve. The above formula was adopted by Brandt (1960) to describe the rotation law of a galaxy represented by a sequence of flattened spheroid. If the mean motions are symmetric with respect to the galaxy rotation axis, every rotation curve observed at a position angle $\phi$ will be described by

$v(r, \phi)=v(r) \cdot P\left(i, \phi-\phi_{\mathrm{n}}\right)$.

Here, $P\left(i, \phi-\phi_{\mathrm{n}}\right)$ is the projection factor, equal to:

$P\left(i, \phi-\phi_{\mathrm{n}}\right)=\frac{\sqrt{\cos ^{2}\left(\phi-\phi_{\mathrm{n}}\right)+\sin ^{2}\left(\phi-\phi_{\mathrm{n}}\right) \cdot \cos ^{-2} i}}{\sin i \cdot \cos \left(\phi-\phi_{\mathrm{n}}\right)}$

Actually, the observed stellar rotation curves should differ from the intrinsic rotation law, because of the asymmetric drift and the integration of the stellar light along the line of sight. However, if the velocity field and the light distributions are symmetric with respect to the galaxy's axis, these effects also must be axially symmetric. In our case, we expect that the bar will generate an asymmetry of the potential that should reflect itself on the orbits as much as the bar is strong. On the other side, differently 
from gas, stellar orbits tend to fill all the energy levels in the space phase, and this may minimize the deviations.

We assumed then for every galaxy a mean rotation curve along the line-of-sight, as described above and we projected it at all the PAs $\phi$ corresponding to our spectra. The disagreement between this circular rotation and the observed curves give us an indication of the noncircular motions present in the galaxy. For practical use, the Brandt formula was rewritten in function of two observable quantities: the maximum rotational velocity $V_{\mathrm{m}}$ and the radius $r_{\mathrm{m}}$ at which this velocity is reached. The parametric expression of the observed rotation curves is then:

$v(r, \phi)=3 V_{\mathrm{m}} \frac{r / r_{\mathrm{m}}}{1+2\left(r / r_{\mathrm{m}}\right)^{3 / 2}} \cdot P\left(i, \phi-\phi_{\mathrm{n}}\right)$.

The model curves were then fitted to the observed ones, deducing $V_{\mathrm{m}}, r_{\mathrm{m}}, \phi_{\mathrm{n}}$ and $i$. They are represented in figures from 2 to 7 as full lines, while the observed data are represented by full squares. The galaxy inclination was deduced also in different way, from the observed axial ratio of the disk, measured at $25 \mathrm{mag} / \operatorname{arcsec}^{2}$, reported in RC3 (de Vaucouleurs et al. 1991) and assuming an intrinsic axial ratio 0.25 (see Table 2). Also the line-of-nodes was, as a first guess, assumed to be coincident with the PA of the galaxy's major axis, according to the idea that the intrinsic structure of the disk is oblate. The derived properties under the assumption of circular motions are reported in Table 3 . The angle $\psi$ between the bar axis and the line-of-nodes is reported in Table 3 , while the intrinsic bar diameter $D_{\circ}$ is reported in Table 2 .

Looking at the two tables, we can see that the differences between the inclination deduced in photometric way (Table 2) and that obtained by fitting the mean rotation (Table 3 ) do not differ more than $13^{\circ}$, a probable random deviation taking into account the uncertainties on the observed and intrinsic axial ratios of the galaxy. Also the angle $\phi_{\mathrm{n}}$ do not differs more than $5^{\circ}$ from the PA of the apparent galaxy major axis, giving support to the hypothesis of oblateness (axial symmetry) of the sample galaxies. The only exception is NGC 6684, where a deviation of $24^{\circ}$ must be attributed to the presence of a triaxial bulge (Bettoni \& Galletta 1988).

The existence of non-circular motions can be checked by means of the circular velocity represented by the model fit and the true velocities observed at different position angles. According to the tests made by Kormendy (1982b) in the case of NGC 936, we considered the following parameters: 1) the presence of deviations with respect to the systemic velocity along the disk minor axis; 2) the ratio $\Delta V_{\text {obs }} / V_{*}$. The parameter $\Delta V_{\text {obs }}$ represents the velocity difference between the major axis rotation curve, projected on the bar position angle, and the bar velocity curve. $V_{*}$ is the projected circular velocity expected along the bar. Positive values of both tests indicate the presence of elongated orbits.
In the first test, we found velocity deviations, in some case quite irregular, along the minor axes of seven galaxies (see figures). Among the remaining cases, NGC 2217, NGC 4267, NGC 4371, NGC 4643 and NGC 6684 exhibit bars close to the apparent major axis (as indicated by the angle $\psi$ in Table 3 ) and the observed motions reflect the stellar motions inside the bars. Only NGC 4754 does not show appreciable deviations from systemic velocity along its minor axis.

The result of the second test is indicated in Table 3 . Most part of deviations are due to the waving pattern presents along the bar, indicated in the last column of the table and described in the previous Sect. 3.2. The amount of the remaining deviation from circular motions is from $10 \%$ to $20 \%$. Only NGC 4371 , with a bar coincident with the galaxy minor axis, does not show deviations. Finally, in the almost edge-on galaxy NGC 4442 the predominant deviation is caused by a cylindric rotation, with equal velocities at different heights from the equatorial plane.

In conclusion, the stellar velocity field shows, in the bar region, deviations from circular velocities lower than $20 \%$. In the outer regions and outside of the bar, the motions seems to be circular.

Acknowledgements. This work has been partially supported by the grant "Astrofisica e Fisica Cosmica" Fondi $40 \%$ of the Italian Ministry of University and Scientific and Technologic Research (MURST).

\section{References}

Bertola F., Bettoni D., Rusconi L., Sedmack G., 1984, AJ 89, 356

Bettoni D., 1989, AJ 97, 79

Bettoni D., Buson L., 1987, A\&AS 67, 341

Bettoni D., Galletta G., 1988, A\&A 190, 52

Bettoni D., Galletta G., 1989, The Messenger 54, 51

Bettoni D., Galletta G., 1994, A\&A 281, 1

Bettoni D., Galletta G., Oosterloo T., 1991, MNRAS 248, 544

Bettoni D., Galletta G., Vallenari A., 1988, A\&A 197, 69

Bettoni D., Galletta G., Sage L.J., 1993, A\&A 280, 121

Brandt J.C., 1960, ApJ 131, 293

Bregman J.N., Hogg D.E., Roberts M.S., 1992, ApJ 387, 484 de Vaucouleurs G., de Vaucouleurs A., Corwin H.G.Jr., Buta R.J., Paturel G., Fouqué P., 1991, Third Reference Catalogue of Bright Galaxies. Springer-Verlag: NewYork (RC3)

Friedly D., Benz W., 1993, A\&A 268, 65

Galletta G., 1987, ApJ 318, 531

Galletta G., 1996, in: Barred Galaxies, IAU Coll.117, Buta R., Crocker D.A. and Elmegreen B.G. (eds.) ASP Conf. Ser. 91,429

Garcia A.M., 1993, A\&AS 100, 47

Jarvis B.J., Dubath P., Martinet L., Bacon R., 1988, A\&AS 74,513

Huntley J.M., 1978, ApJ 225, L101

Kent S.M., 1990, AJ 100, 377

Kormendy J., 1982a, ApJ 257, 75 
Kormendy J., 1982b, in: Morphology and Dynamics of Galaxies, XX Adv. Course Saas-Fee, Martinet L. \& Major L. (eds.) p. 115

Kormendy J., 1983, ApJ 275, 529

Magrelli G., Bettoni D., Galletta G., 1992, MNRAS 256, 500

McElroy D.B., 1995, ApJS 100, 105

Peterson C.J., Huntley J.M., 1980, ApJ 242, 913

Peterson C.J., Rubin V.C., Ford W.K., Thonnard N., 1978, ApJ 219, 31

Reduzzi L., Rampazzo R., 1995, Ap. Let. Comm. 30, 1
Roberts M.S., Hogg D.E., Bregman J.N., Forman W.R., Jones C., 1991, ApJS 75, 751

Sandage A., Bedke J., 1995, The Carnegie Atlas of Galaxies, Carnegie Institution of Washington, Washington DC, RSA

Sellwood J.A., Wilkinson A., 1993, Rep. Prog. Phys. 56, 173

Veron-Cetty M.P., Veron P., 1986, A\&AS 66, 335

Wozniak H., Pfenniger D., 1996, in: Barred Galaxies, IAU Coll.117, Buta R., Crocker D.A. and Elmegreen B.G. (eds.), ASP Conf. Ser. 91, 445 